J. Dairy Sci. 96:4771-4771

http://dx.doi.org/10.3168/jds.2013-96-7-4771

(c) American Dairy Science Association ${ }^{\circledR}, 2013$.

\title{
Erratum to "Reliabilities of genomic prediction using combined reference data of the Nordic Red dairy cattle populations" (J. Dairy Sci. 94:4700-4707)
}

\author{
R. F. Brøndum, E. Rius-Vilarrasa, I. Strandén, G. Su, B. Guldbrandtsen, W. F. Fikse, and M. S. Lund
}

Table 5 (page 4706) contained incorrect data in column "FIN" of the combined Danish-Swedish-Finnish (DK-SWE-FIN) reference data set. The corrected table is shown below (corrected data in bold).

The authors regret the error.

\section{REFERENCES}

Brøndum, R. F., E. Rius-Vilarrasa, I. Strandén, G. Su, B. Guldbrandtsen, W. F. Fikse, and M. S. Lund. 2010. Reliabilities of genomic prediction using combined reference data of the Nordic Red dairy cattle populations. J. Dairy Sci. 94(9):4700-4707.

Table 5. Reliabilities of genomic selection for combined Swedish-Finnish (SWE-FIN) reference and combined Danish-Swedish-Finnish (DK-SWE-FIN) reference data ${ }^{1}$

\begin{tabular}{|c|c|c|c|c|c|c|c|}
\hline \multirow[b]{2}{*}{ Item } & \multicolumn{3}{|c|}{ Reference: SWE-FIN } & \multicolumn{4}{|c|}{ Reference: DK-SWE-FIN } \\
\hline & SWE & FIN & Both & DK & SWE & FIN & All \\
\hline Size of reference & 2,986 & 2,986 & 2,986 & 3,735 & 3,735 & 3,735 & 3,735 \\
\hline Size of test & 1,411 & 1,575 & 2,986 & 778 & 1,395 & 1,562 & 3,735 \\
\hline Milk yield & 0.21 & 0.29 & 0.26 & 0.25 & 0.23 & 0.29 & 0.27 \\
\hline Fat yield & 0.26 & 0.37 & 0.32 & 0.31 & 0.30 & 0.38 & 0.34 \\
\hline Protein yield & 0.20 & 0.25 & 0.23 & 0.18 & 0.21 & 0.24 & 0.22 \\
\hline Yield index & 0.22 & 0.27 & 0.25 & 0.18 & 0.23 & 0.26 & 0.23 \\
\hline Udder health & 0.25 & 0.29 & 0.28 & 0.23 & 0.25 & 0.29 & 0.28 \\
\hline Fertility & 0.25 & 0.25 & 0.27 & 0.21 & 0.27 & 0.24 & 0.26 \\
\hline Other diseases & 0.30 & 0.31 & 0.34 & 0.47 & 0.34 & 0.31 & 0.43 \\
\hline Longevity & 0.11 & 0.20 & 0.16 & 0.15 & 0.10 & 0.20 & 0.15 \\
\hline Direct calving & 0.25 & 0.37 & 0.32 & 0.28 & 0.27 & 0.38 & 0.32 \\
\hline Maternal calving & 0.09 & 0.20 & 0.23 & 0.29 & 0.09 & 0.21 & 0.26 \\
\hline Body & 0.38 & 0.14 & 0.41 & 0.38 & 0.41 & 0.36 & 0.55 \\
\hline Feet and legs & 0.34 & 0.28 & 0.24 & 0.30 & 0.37 & 0.14 & 0.31 \\
\hline Udder conformation & 0.26 & 0.31 & 0.29 & 0.27 & 0.29 & 0.29 & 0.38 \\
\hline Milking ability & 0.25 & 0.26 & 0.29 & 0.18 & 0.29 & 0.32 & 0.29 \\
\hline Temperament & 0.33 & 0.18 & 0.29 & 0.33 & 0.35 & 0.26 & 0.33 \\
\hline Nordic total merit index & 0.20 & 0.14 & 0.21 & 0.19 & 0.18 & 0.16 & 0.19 \\
\hline Growth & 0.36 & 0.17 & 0.36 & 0.29 & 0.32 & 0.15 & 0.32 \\
\hline Mean (5-fold) & 0.25 & 0.27 & 0.28 & 0.26 & 0.26 & 0.26 & 0.30 \\
\hline Mean (young) & 0.26 & 0.25 & 0.26 & 0.23 & 0.27 & 0.26 & 0.28 \\
\hline
\end{tabular}

${ }^{1}$ Reliabilities are estimated for Danish (DK), Swedish (SWE), Finnish (FIN), Swedish-Finnish (both), and Danish-Swedish-Finnish (all) animals. Mean reliability is also given using only the youngest cross-validation group. The standard deviation of reliabilities between the 5 cross-validation test data sets ranged between 0.02 and 0.07 . 